\title{
Cytoskeletal tropomyosins: choreographers of actin filament functional diversity
}

\author{
Howard Vindin · Peter Gunning
}

Received: 21 March 2013/Accepted: 9 July 2013/Published online: 1 August 2013

(C) The Author(s) 2013. This article is published with open access at Springerlink.com

\begin{abstract}
The actin cytoskeleton plays a central role in many essential cellular processes. Its involvement requires actin filaments to form multiple populations with different structural and therefore functional properties in specific subcellular locations. This diversity is facilitated through the interaction between actin and a number of actin binding proteins. One family of proteins, the tropomyosins, are absolutely essential in regulating actin's ability to form such diverse structures. In this review we integrate studies from different organisms and cell types in an attempt to provide a unifying view of tropomyosin dependent regulation of the actin cytoskeleton.
\end{abstract}

Keywords Tropomyosin - Cytoskeleton - Actin · Cytoskeletal regulation

$\begin{array}{ll}\text { Abbreviations } \\ \text { HMW } & \text { High molecular weight } \\ \text { LMW } & \text { Low molecular weight } \\ \text { ABPs } & \text { Actin binding proteins }\end{array}$

\section{Introduction}

The actin cytoskeleton is a diverse system involved in a plethora of cellular functions including adhesion, cytokinesis, cell motility, contractile force, signaling, intracellular transport and apoptosis. There is now a mounting body of evidence demonstrating that the ability of one filament system to perform such a remarkable range of functions is

H. Vindin · P. Gunning $(\bowtie)$

Oncology Research Unit, School of Medical Sciences,

University of New South Wales, Sydney, NSW, Australia

e-mail: p.gunning@unsw.edu.au facilitated through the functional specification of actin filaments by their associated tropomyosin isoform(s) (Gunning et al. 2005). Historically tropomyosin has been referred to as muscle or non-muscle. However, muscle has been shown to express tropomyosin localized to the actin cytoskeleton that is distinct from those present in the contractile apparatus (Kee et al. 2009). Therefore, isoforms present in the contractile apparatus of muscle will be referred to as muscle tropomyosin, whilst cytoskeletal tropomyosin will be used to describe the isoforms present in the cytoskeleton of all cells.

In mammalian cells tropomyosin is encoded by four genes, TPM1, 2, 3 and 4, which through use of multiple promoters and alternative splicing of exons lead to the expression of over 40 isoforms (Pittenger et al. 1994; Dufour et al. 1998; Cooley and Bergtrom 2001). These have historically been classified as either high molecular weight (HMW) ( 284 amino acids) or low molecular weight (LMW) ( $\sim 248$ amino acids), that correspond to the use of either exons 1 a plus 2 or exon $1 \mathrm{~b}$ respectively to encode their N-termini (Pittenger et al. 1994). The molecular diversity seen in tropomyosin isoforms comes from the substantial differences seen in alternatively spliced exons (Fig. 1) (Schevzov et al. 2011).

The specialized contractile systems of striated and smooth muscle utilize a total of four actin and only five tropomyosin isoforms (Herman 1993; Pittenger et al. 1994). In contrast, there are just two cytoskeletal actin isoforms and over 40 tropomyosin isoforms found in the cytoskeleton of mammalian cells which facilitate the functional diversity seen in the actin filament system of the cytoskeleton (Gunning et al. 2005, 2008). The role of tropomyosin has been extensively reviewed in Gunning et al. (2005, 2008). In this article we integrate the genetic, molecular cell biology and protein chemistry studies to 
Fig. 1 Diagram of the TPM1 $(\alpha)$, TPM2 $(\beta)$, TPM3 $(\gamma)$ and TPM4 $(\delta)$ genes and the isoforms they encode. The white boxes show untranslated regions, dotted lines represent introns and the black boxes show exons common to all isoforms. Muscle isoforms (shown highlighted in red) account for only five tropomyosin isoforms expressed in mammalian cells. Only the major isoforms are included, a number of mRNAs have been detected only by RT-PCR and are not shown
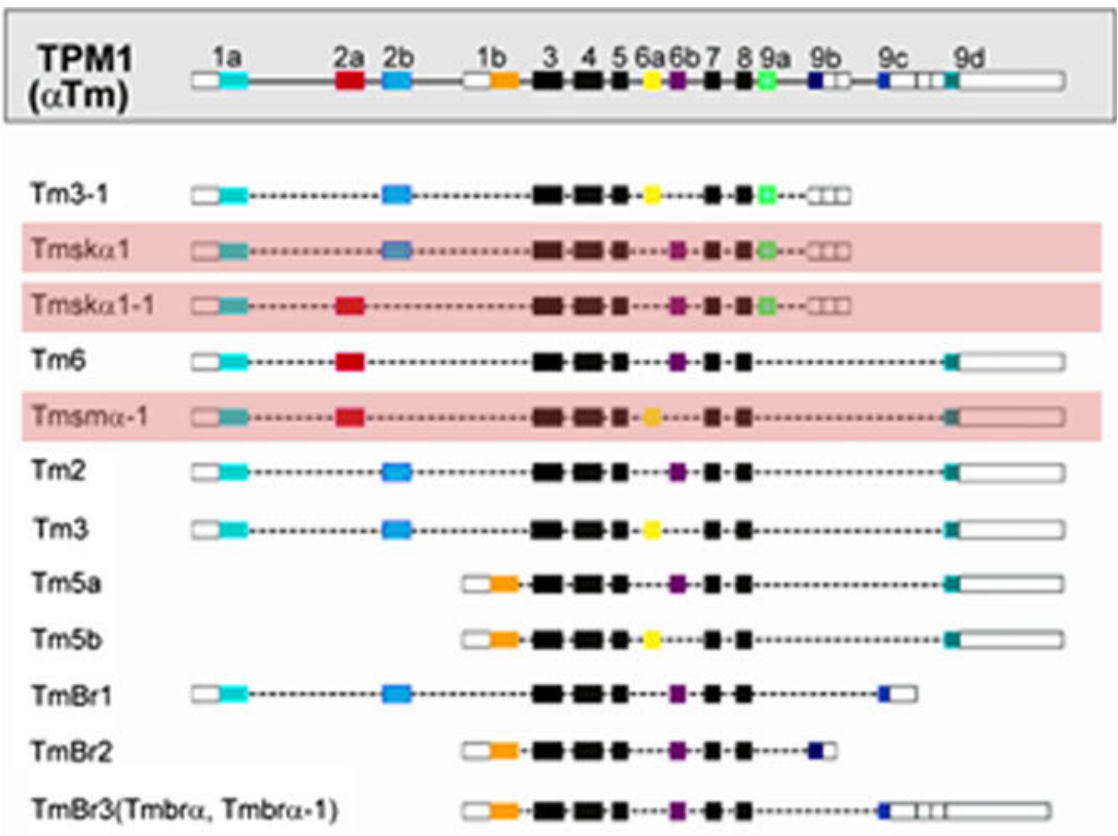

TPM2
$(\beta T m)$$\quad$ a $\quad 2 b \quad 3456 a 6 b 89 a \quad 9 d$

\begin{tabular}{|c|c|}
\hline hTmskß & 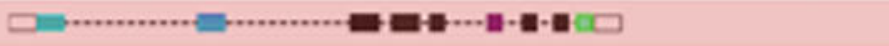 \\
\hline Tm1 & 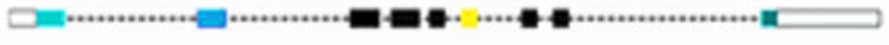 \\
\hline $\begin{array}{c}\text { TPM3 } \\
(\gamma \mathrm{Tm})\end{array}$ & $2 b \quad 1 b-3.45606789 a 969 c-9 d$ \\
\hline
\end{tabular}

\begin{tabular}{|c|c|}
\hline$a_{g} T m 1$ & 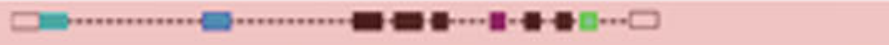 \\
\hline Tm5NM1 & $\square=-\left[\begin{array}{l}0 \\
0\end{array}\right.$ \\
\hline Tm5NM2 & ben- \\
\hline Tm5NMS &  \\
\hline Tm5NM6 & 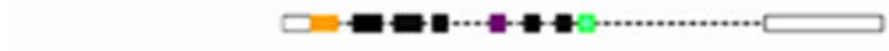 \\
\hline TmSNM4 & 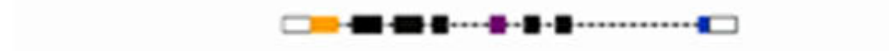 \\
\hline Tm5NM7 & $\square=0$ \\
\hline Tm5NM8 & 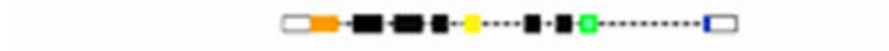 \\
\hline Tm5NM9 & $\square=0$ \\
\hline Tm5NM10 & $\square=\operatorname{ne-~}$ \\
\hline Tm5NM11 & 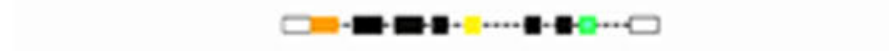 \\
\hline $\operatorname{Tm} 5 \mathrm{NM} 3$ & 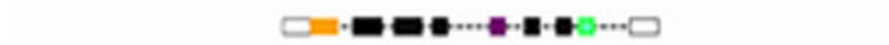 \\
\hline
\end{tabular}

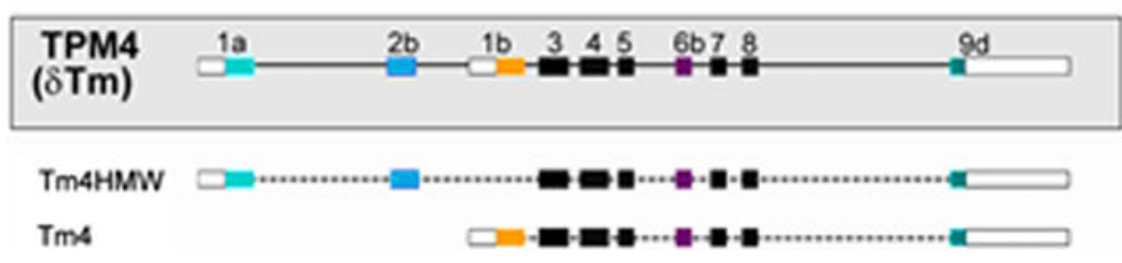


provide a unifying view of how tropomyosin isoforms act as choreographers of the diversity of function of the animal actin cytoskeleton.

We initially cover the experiments which establish the lack of functional redundancy between tropomyosin isoforms. This leads to consideration of the intracellular spatial segregation of tropomyosin isoforms which provide evidence that these isoforms perform spatially and functionally distinct roles in the cell. The intracellular functional specificity of tropomyosin isoforms is examined in a range of cell types which leads to the conclusion that the spatial segregation of tropomyosins has driven the evolution of functional specialisation. Mechanisms of functional specialisation are then covered with respect to isoform specific interactions with actin binding proteins. Finally, it is proposed that for actin filaments containing tropomyosin, it is the actin-tropomyosin co-polymer which should be considered as the unit of function.

\section{Tropomyosins are not functionally redundant}

Tropomyosin is essential in yeast

The first work highlighting the essential nature of cytoskeletal tropomyosins was performed in Schizosaccharomyces pombe (Balasubramanian et al. 1992). They found that haploid spores carrying the disrupted allele were still able to germinate, though they die soon after as elongated single cells (Balasubramanian et al. 1992). This study also highlighted the essential role that the Cdc8 product, the only tropomyosin isoform present in S. Pombe, has in generating the contractile ring required for cytokinesis.

In Saccharomyces cerevisiae two tropomyosin isoforms are present; Tpm1p and Tpm2p which are encoded by the TPM1 and TPM2 genes respectively (Drees et al. 1995). Previous work by Liu and Bretscher (1989) had shown that whilst not lethal, the disruption of TPM1 gene expression results in both the disappearance of actin cables and a reduced growth rate. Interestingly whilst a loss of TPM2 gene expression shows no detectable phenotype, disruption of both TPM1 and TPM2 expression results in lethality. This illustrates that in yeast, the expression of at least one isoform is essential for cell viability (Drees et al. 1995). Furthermore it was shown that an elevated expression of the TPM2 gene could not compensate for the loss of TPM1 expression in $S$. cerevisiae, providing evidence for their functional differences (Drees et al. 1995). These results illustrate that in yeast, tropomyosin is essential for cell survival and in the case of $S$. cerevisiae, one isoform performs a specific function that overexpression of the other cannot compensate for.
Mammalian tropomyosin genes are not redundant and perform essential functions

TPM1 ( $\alpha$-) and TPM2 ( $\beta$-tropomyosin) genes

Homozygous knockout of the TPM1 gene in mice results in embryonic lethality between embryonic day 8.5 and 11.5 (Rethinasamy et al. 1998). Furthermore, it was shown using heterozygous knockout mice that despite a $50 \%$ decrease in mRNA from striated muscle $\alpha$-Tm in the heart there was no difference in total $\alpha$-Tm or compensation from other isoforms demonstrated by unaltered levels of the $\beta$-Tm protein between heterozygous and control littermates (Rethinasamy et al. 1998). A separate study found that knockout of only the $\alpha$-Tm striated muscle isoform also results in embryonic lethality, however this occurred between embryonic day 9.5 and 13.5 (Blanchard et al. 1997). These results when taken together suggest that the $\alpha$-Tm striated muscle isoform and one or more other isoforms encoded for by the TPM1 gene are critical for at least two essential processes in embryonic development. Further work by Wieczorek's laboratory using transgenic mice demonstrated that changes in relative levels of skeletal tropomyosin in the heart by exchanging striated muscle $\beta$-Tm with striated muscle $\alpha$-Tm does not change the total tropomyosin expression, however the ectopic expression of $\beta$-Tm causes severe cardiac pathological abnormalities (Muthuchamy et al. 1995, 1998; Palmiter et al. 1996). This suggests that different tropomyosin isoforms can confer different structural/functional information onto the actin filaments they bind to, allowing them to perform specific functions, and in the heart muscle only striated muscle $\alpha$-Tm is able to provide the structural/functional information essential for normal cardiac function. It has also been observed that homozygous knockout of the TPM2 gene results in a failure in early developmental processes (Jagatheesan et al. 2010).

\section{TPM3 ( $\gamma$-tropomyosin) gene}

Knockout of the TPM3 gene which encodes for 11 cytoskeletal isoforms (Tm5NM1-11) has also been shown to be embryonically lethal in mice by embryonic day 2.5 indicating that at least one LMW product from this gene is essential very early in embryonic development (Hook et al. 2004). This data taken with that from the TPM1 and TPM2 genes illustrates that these genes are not functionally redundant and each is essential for survival. This study also demonstrated that at least the TPM3 gene is essential for embryonic stem cell viability. Given that all four tropomyosin genes are expressed in both embryos and embryonic stem cells, this data demonstrates that the loss in 
viability is due to the essential functions fulfilled by isoforms from this gene. Further work on the TPM3 gene demonstrated that whilst the deletion of exon 9d-containing isoforms Tm5NM1 and Tm5NM2 lead to partial embryonic lethality in mice, the deletion of the exon 9c-containing isoforms Tm5NM4 and Tm5NM7 does not affect embryonic development. This shows that exon 9d-containing isoforms cannot fully be compensated for by other tropomyosin isoforms in embryonic development (Hook et al. 2011). Whilst the absence of lethality in the exon 9c knockout mice may indicate there is some intragenetic functional redundancy in embryonic development, this does not preclude these isoforms from fulfilling essential functions in later life, or perhaps their function in development may be to help regulate actin filament function in the event of a failure to express other isoforms from the TPM3 gene. Interestingly, deletion of Tm5NM1/2 in stem cells yields no viable stem cells indicating that this subset of isoforms performs at least one essential function (Hook et al. 2011).

\section{Conclusion}

This data provides strong evidence for the idea that multiple tropomyosin isoforms are critical for cell survival. The work performed by Weiczorek's laboratory using striated muscle $\beta-T m$ transgenic mice further demonstrates that the loss or exchange of one isoform for another leads to different functional properties (Palmiter et al. 1996). The lack of redundancy seen between the mammalian genes suggests that these isoforms have different functional properties and that these are required for essential processes in both embryonic development and the maintenance of cellular processes in later life. As a whole these studies support the notion that the regulation of distinct populations of actin filaments by specific tropomyosin isoforms provides a mechanism to fulfill the wide range of specific cellular functions required of the actin cytoskeleton.

\section{Cytoskeletal tropomyosin isoforms are spatially segregated}

\section{Introduction}

The first reports which suggested that tropomyosin isoforms are present at different intracellular locations were by Burgoyne and Norman (Burgoyne and Norman 1985a, b). They first observed that in adrenal chromaffin cells, which express three different tropomyosin isoforms, only one of these was seen to associate with chromaffin granule membranes, suggesting that this specific isoform may be involved in vesicle transport or tethering (Burgoyne and
Norman 1985b). They also observed that in neurons, tropomyosins were enriched in cell bodies and dendrites compared to the axons. Thus the nature and composition of the cytoskeletal structures present in the axon and dendrites may differ (Burgoyne and Norman 1985a) and this has been subsequently confirmed in multiple studies (for review, see Gunning et al. 1998a).

Lin et al. (1988) were the first to directly visualize the spatial segregation of isoforms. They reported that whilst both HMW and LMW tropomyosins were seen in stress fibers, only the LMW isoforms were present in ruffling membranes (Lin et al. 1988). These studies have been repeated in a number of cell types and with the ability to see increasing detail and differentiate between more isoforms there has been an increased realization of the extent to which these isoforms are spatially segregated.

\section{Experimental approaches to isoform sorting}

Five independent approaches to isoform sorting have been used by multiple groups to address the question of cytoskeletal-tropomyosin isoform sorting. While there are potential weaknesses with each approach in isolation, the concordance of the multiple approaches has provided confidence that the intracellular sorting of tropomyosin isoforms is an absolute intrinsic property of tropomyosins in all cellular systems in which this has been studied.

Antibodies have been the most widely used tools to approach the sorting of tropomyosin isoforms. The most widely used antibodies have been generated by the Lin and Gunning groups using two completely different strategies. Recent evaluation of the specificity of all the available antibodies from these two groups using panels of purified tropomyosin isoforms has demonstrated that they show a remarkable level of specificity and has also highlighted where care must be taken with potential cross-reactivity (Schevzov et al. 2011). There are now multiple antibodies (polyclonal and monoclonal) available for most isoforms which are used to provide confirmation of results. The biggest concern with antibody studies is the potential of epitope masking due to local conformation changes or binding of associated proteins which obscure the epitope. While antigen retrieval can address this in some situations, it cannot provide absolute certainty that an isoform is absent.

The use of tagged-tropomyosins, usually GFP (or related colours)-derivatives has been widely used to locate and follow individual isoforms. This is most powerful when used in conjunction with antibody staining such as the very careful work of both Temm-Grove et al. (1998) and Tojkander et al. (2011). The combination of both approaches provides very compelling evidence for the specificity of isoform sorting. 
Similarly, the use of in situ hybridization to localize specific tropomyosin isoform mRNAs has provided compelling evidence for the intracellular sorting of tropomyosins. Hannan et al. $(1995,1998)$ demonstrated isoform specific localization of tropomyosin isoform mRNAs which was related to the localization of the corresponding proteins revealed by isoform-specific antibodies. While there was not a one-to-one correspondence of mRNA and protein in neurons in vivo and in vitro, there was a clear concordance of mRNA and protein polarity.

Biochemical sub-fractionation has been used in some cases to detect specific tropomyosins associated with specific intracellular structures/compartments. This was originally used by Burgoyne and Norman (1985b) to demonstrate the association of specific tropomyosins with cromaffin granules and has also been used to confirm the presence of Tm5NM1/2 with Golgi-derived structures (Heimann et al. 1999). The use of sub-fractionation also brings with it potential problems of contamination but is powerful when combined with the other approaches.

Finally, gene knockout or siRNA knockdown experiments have been used to show that removal of the isoform removes antibody staining and/or impacts the function of the compartment containing the isoform. Loss of function can be problematic because of rescue by another isoform but where loss of function is seen, it is most compelling.

Below we consider the wealth of experimental systems and approaches which have unambiguously established the generality of tropomyosin isoform intracellular sorting. The studies documented below have used a range of different approaches or have been confirmed in multiple labs; often using different antibodies or different approaches.

\section{Differential sorting of Cdc8p in yeast}

In fission yeast, the acetylation of the only tropomyosin isoform expressed; $\mathrm{Cdc} 8 \mathrm{p}$ has a significant impact on its ability to bind and regulate actin filaments (Skoumpla et al. 2007). Coulton et al. (2010) found that acetylated Cdc8p was strongly associated with actin filament bundles in the cytokinetic actomyosin ring (CAR). In contrast, unacetylated Cdc8p was never seen within the CAR and was only associated with filament bundles that extend throughout the cell (Fig. 2a). Since tropomyosins have not been found in either plants or amoebae (Pruyne 2008) these observations demonstrate that sorting tropomyosin isoforms is an intrinsic property that is as old as tropomyosin itself.

\section{Neurons}

Had et al. (1994) compared the localization of two isoforms $\mathrm{Tm} 4$ and $\mathrm{TmBr} 3$ both in cultured neurons and in the mouse. They found that $\mathrm{Tm} 4$ was concentrated at the
A

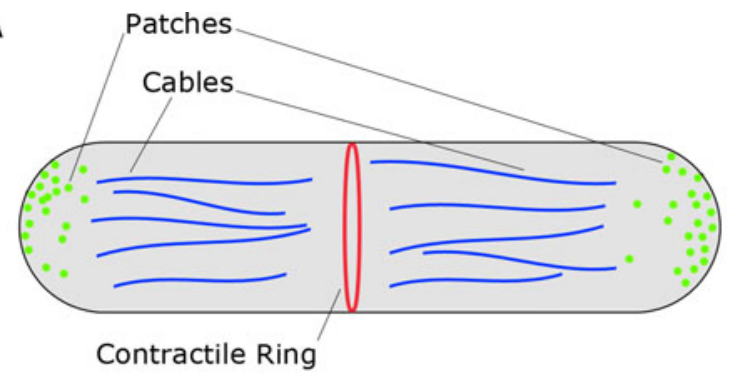

B

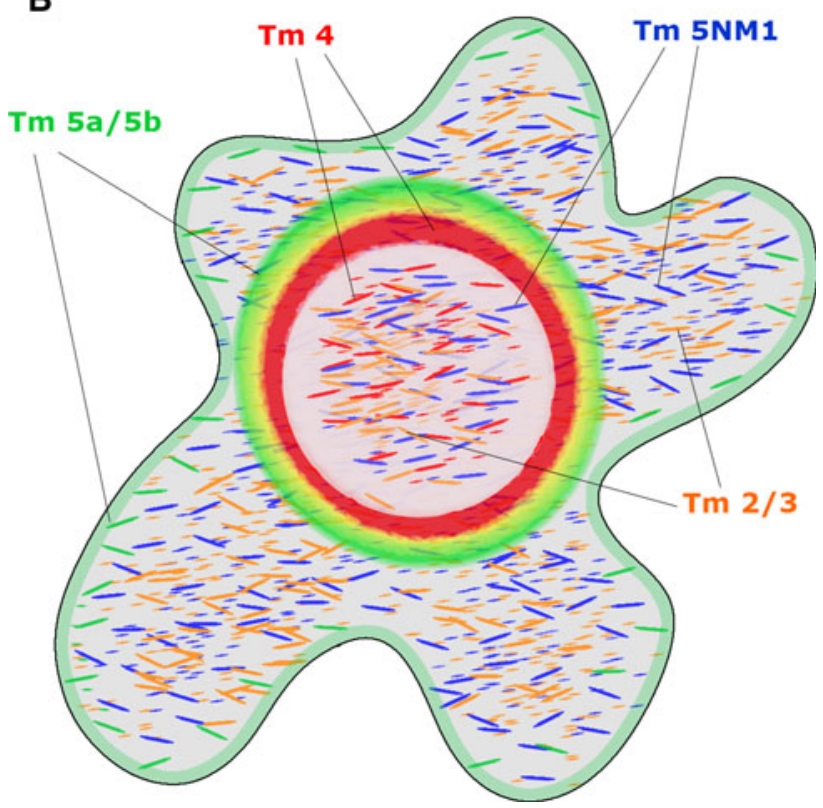

Fig. 2 a Distribution of tropomyosin in Schizosaccharomyces pombe. Actin patches are found near the cell periphery and are not associated with tropomyosin isoforms. The cables which run throughout the cell are associated with unacetylated Cdc8p and favour the binding of myosin-V. In contrast, the contractile ring actin is associated with acetylated $\mathrm{Cdc} 8 \mathrm{p}$ which favours the binding of myosin-II. b Distribution of tropomyosin in Osteoclasts plated on ivory. Tm4 (red) is associated with podosomes (represented as the inner ring) and the interior of the cell. Tm5a/5b (green) is associated with the F-actin ring (represented as the outer ring) and is slightly enriched near the plasma membrane. Whilst some colocalization is observed between $\mathrm{Tm} 5 \mathrm{a} / 5 \mathrm{~b}$ and $\mathrm{Tm} 4$ (yellow), Tm5a/5b are notably absent from the podosomes. Both Tm $2 / 3$ (orange) and Tm5NM1 (blue) are both found throughout the cell in different subcellular pools, however the nature of these regions is not yet known

growth cones of neurons whereas $\mathrm{TmBr} 3$ was notably absent from these regions. In vivo, Tm4 was restricted to postsynaptic regions whilst $\mathrm{TmBr} 3$ was concentrated at presynaptic sites suggesting that these isoforms fulfill different functional roles in neurons (Had et al. 1994). This spatial segregation has also been detected during neuronal development. In developing neurons Tm5NM1/2 is restricted to the developing axons. However in mature neurons its localization is somatodendritic, and its loss from the axon occurs coincident with the initial appearance 
of TmBr3 in axons (Weinberger et al. 1996). This temporal regulation has also been seen with $T m 5 \mathrm{a} / 5 \mathrm{~b}$ where its presence in the growth cones of primary neurons diminishes with time in culture (Schevzov et al. 1997).

Further experiments revealed that depolymerization of actin filaments through the addition of cytochalasin B resulted in a loss of spatial segregation of Tm5NM1/2. After wash-out of the drug the spatial segregation of tropomyosin isoforms was restored and Tm5NM1/2 was again mostly absent from the growth cone (Schevzov et al. 1997). This clearly demonstrates that the tropomyosin isoform composition of actin filaments is dependent on the dynamic remodeling of the cytoskeleton.

\section{Osteoclasts}

Osteoclasts possess a highly dynamic cytoskeleton capable of forming a number of distinct intracellular structures which can be defined by the localization of specific tropomyosin isoforms. At the podosomal attachment structures, both Tm4 and Tm5a/5b are present although their localization on these structures is mutually exclusive (McMichael et al. 2006). Whilst Tm4 is found to associate with the interior ends of podosomal actin cores and the top half of F-actin rings in these cells, Tm5a/5b was enriched at the base of podosomal cores and the outer edge of the F-actin rings (McMichael et al. 2006). In contrast, staining for Tm5NM1 and Tm2/3 showed that these isoforms are excluded from attachment structures. Despite both these isoforms being enriched in the cell interior, there was little to no overlap between them (Fig. 2b). It was concluded that they are localized to distinct internal structures in these cells (McMichael et al. 2006). These results indicate that in osteoclasts there are at least four cytoskeletal structures which are associated with specific tropomyosin isoforms.

\section{Skeletal muscles}

Muscle fibers contain three skeletal muscle tropomyosin isoforms which form part of the thin filament where they are involved in the regulation of muscle contraction (Huxley 1973). In addition to skeletal tropomyosin, two cytoskeletal isoforms are expressed which sort to specific compartments within the myofibril. Within the myofibril, Tm5NM1 is specifically sorted to both a filament network adjacent to the Z-line and a subsarcolemma filament system found around the periphery of the myofibril (Kee et al. 2004). Tm4 is also expressed in muscle fibers where it is sorted to two specific locations. Like Tm5NM1, this isoform is sorted to a filament network adjacent to the Z-line where these two isoforms define distinct actin filament populations (Vlahovich et al. 2009). Tm4 is also localized to longitudinal filaments running perpendicular to the
Z-line which are associated with muscle fibers undergoing remodeling and repair (Vlahovich et al. 2008).

Smooth muscle cells

More recently it was found that at least five tropomyosin isoforms are expressed in vascular smooth muscle cells. In addition to the smooth muscle tropomyosin isoforms Tm1 and Tm6 three cytoskeletal isoforms Tm2, Tm5NM1 and Tm4 were also present (Gallant et al. 2011). In contrast to previous work on chicken gizzard smooth muscle by Sanders et al. (1986) in which heterodimer formation was observed, not only did Tm1 and Tm6 not form heterodimers in this cell type but these isoforms also sorted to different intracellular regions and were associated with different actin isoforms (Gallant et al. 2011).

\section{Fibroblasts}

Work in NIH 3 T3 fibroblasts also demonstrated that specific tropomyosin isoforms are differentially sorted to specific subcellular locations (Percival et al. 2000). One hour after replating, products from the TPM1 gene were incorporated into stress fiber structures, whereas those from the TPM3 gene were localized to the perinuclear region. This sorting becomes less distinct as the cells progress through the cell cycle. After $8 \mathrm{~h}$ isoforms from both genes were localized in stress fibers, however TPM3 isoforms were still present in the central cytoplasm and TPM1 isoforms were more enriched at the cell periphery (Percival et al. 2000). It was later shown that Tm5NM2 specifically sorted to short actin filaments associated with the Golgi complex (Percival et al. 2004).

In primary mouse embryo fibroblasts similar spatial segregation is also seen. Schevzov and colleagues found that the HMW isoforms from the TPM1 gene sorted predominately to stress fibers, whilst Tm5a/5b were the only isoforms specifically located to the ruffling membranes (Schevzov et al. 2005b, 2011).

\section{Tropomyosin isoforms sort within stress fibers}

More recently it has been shown that individual tropomyosin isoforms are further segregated into specific regions along stress fibers. Tojkander et al. (2011) found that only $\mathrm{Tm} 2$ was localized along entire stress fibers whilst Tm1, Tm5NM1 and Tm5NM2 were concentrated at the distal ends of filament bundles corresponding to focal adhesions. $\mathrm{Tm} 3$ and Tm4 were found proximally to focal adhesions, where they were seen either as short segments or as a dotted pattern. Further results using live-cell imaging demonstrate that tropomyosin isoforms are sequentially recruited to both focal adhesions and dorsal stress fibers 
and Tm4's localization to dorsal stress fibers coincides with the incorporation of myosin II into these structures (Tojkander et al. 2011). Furthermore, it was shown that at least four different tropomyosins are required for stress fiber formation (Tojkander et al. 2011).

\section{Mechanism of isoform sorting}

The mechanism of isoform sorting has been the subject of extensive reviews (Gunning et al. 1998a, b, 2005, 2008; Martin and Gunning 2008) which can be summarized very simply. The isoforms are locally assembled and held in place by higher order structures (Martin and Gunning 2008). The site of protein synthesis of isoforms may aid in sorting but does not absolutely determine isoform location (Hannan et al. 1995, 1998). There is no evidence for transport of isoforms to specific intracellular locations (Martin et al. 2010). Hence, the mechanism of sorting most likely occurs at the level of local assembly of the actin filament by mechanism(s) as yet unknown.

\section{Conclusion}

It has been known for years that tropomyosin isoforms are spatially segregated to distinct actin filament populations and this extensive accumulation of data has established that tropomyosin isoform sorting is a fundamental cellular process which is shared across all animal cells.

\section{Cytoskeletal tropomyosin function}

The finely regulated spatial segregation of tropomyosin isoforms is necessary as it ensures that individual isoforms are in the correct locations to fulfill specific functions critical for the normal functioning of the cell.

\section{Neuronal morphogenesis}

Given the fine spatial regulation of tropomyosin isoforms seen in neurons, these cells have been more extensively studied to determine the effects of tropomyosin on neuronal morphogenesis. Primary neurons from transgenic mice overexpressing Tm5NM1 were found to have increased neuronal branching in both dendrites and axons and a significant increase in growth cone size without any noticeable change in its gross morphology (Schevzov et al. 2005a). In contrast, neurons from transgenic mice overexpressing Tm3 had both significantly decreased numbers and length of dendrites. These results indicate that Tm5NM1 and Tm3 contain different structural information, and their expression gives rise to filament populations with different functional properties (Schevzov et al. 2005a). Consistent with the increase in dendritic length seen with Tm5NM1 overexpression, neurons from mice lacking Tm5NM1/2 were seen to have a significant decrease in dendritic length, as well as a number of other morphological changes compared to control neurons (Fath et al. 2010). These results show that altering the tropomyosin composition of filaments in neurons leads to significant changes in neuronal morphogenesis.

\section{Trafficking}

Organelle transport plays an essential part in many cellular functions and is a process which relies on both actin filaments and microtubules. Pelham et al. (1996) investigated the role of tropomyosin in organelle transport through the microinjection of Tm3 into NRK cells. They found that the microinjection of Tm3 but not Tm5NM1 causes a remarkable redistribution of membrane-bound organelles into the perinuclear region (Pelham et al. 1996). These results at the very least indicate that these two isoforms are functionally distinct.

\section{CFTR membrane levels}

The actin cytoskeleton has also been shown to play a role in the delivery of the cystic fibrosis transmembrane conductance regulator (CFTR) into the apical membrane of epithelial cells. It has been shown that reduced expression of Tm5a and Tm5b result in an increased surface expression of CFTR in vitro indicating that these isoforms may be associated with a subpopulation of actin filaments directly involved in the removal of CFTR from the plasma membrane (Dalby-Payne et al. 2003). It was concluded that these isoforms play a role in the regulation of endocytosis.

\section{Cytokinesis}

A number of studies have shown that tropomyosin plays an important part in cytokinesis. In yeast the role that tropomyosin plays in the regulation of cytokinesis has been extensively studied. Balasubramanian et al. (1992) found that the tropomyosin isoform $\mathrm{Cdc} 8$ was essential for cell survival in $S$. pombe. Whilst Cdc8 was not required for spore germination, cell growth or DNA replication it is essential for cytokinesis. This indicates that the essential role for this protein is to form part of the F-actin contractile ring (Balasubramanian et al. 1992). Further work by Mulvihill's laboratory showed that the function and sorting of Cdc8 to different cellular structures was dependent upon its acetylation (Skoumpla et al. 2007; Coulton et al. 2010). The cytokinetic deficit found in cells which lack the NatB $N$ - $\alpha$-acetyltransferase regulatory subunit was also shown to be as a result of a lack of tropomyosin acetylation (Coulton 
et al. 2010). Stark et al. (2010) found that this regulation of cytokinesis in $S$. pombe was through its role in stabilizing actomyosin interactions.

The regulation of cytokinesis by tropomyosin has also been seen in mammalian cells. Hughes et al. (2003) examined tropomyosin expression in developing and neoplastic brain tissue. They found that in the embryonic brain HMW tropomyosin expression was restricted to proliferative areas, whereas in the adult brain, staining could only be seen in blood vessels. They also noted that in rare proliferating astrocytes HMW tropomyosins were found in the contractile ring, but after withdrawal from the cell cycle HMW tropomyosin expression was down regulated (Hughes et al. 2003). Forced expression of Tm5NM1 and a chimeric tropomyosin Tm5/3 in Chinese hamster ovary cells resulted in faster cell division which would suggest that tropomyosin is necessary for the formation of the contractile ring (Eppinga et al. 2006). The abnormal division seen in Tm5NM1 overexpressing cells may be due to the inability of other actin binding proteins to disassemble the contractile ring. This is supported by the observation that Tm5NM1 excludes the association of ADF with filaments containing this tropomyosin isoform (Bryce et al. 2003).

\section{Podosomes in osteoclasts}

Osteoclasts express several cytoskeletal isoforms which sort to specific regions. Tm4 was found to be associated with the core of podosomes, suggesting that it may play a role in regulating these structures (McMichael et al. 2006). Knockdown and overexpression studies revealed a direct role for Tm4 in regulating both podosomal and sealing zone actin filaments. McMichael and Lee (2008) found that either under- or overexpression of Tm4 disrupted these attachment structures leading to impaired bone resorption and cell motility. Further work examining the role of Tm2 and Tm3 in these cells revealed that despite a lack of association with distinct actin structures in these cells, these isoforms play a role in the regulation of osteoclast morphology and function (Kotadiya et al. 2008). These results taken together demonstrate that individual isoforms decorate distinct actin filament populations in osteoclasts, giving them specific functional properties necessary for normal cellular function.

Stem cell viability

A number of tropomyosin isoforms play a critical role in the regulation of stem cell viability and embryonic development. Eliminating the cytoskeletal isoforms from the TPM3 gene (Tm5NM1-11) results in lethality prior to embryonic day 2.5 and the inability to generate viable stem cells (Hook et al. 2004). Embryonic stem cells deleted for exons 9a/9b of the TPM3 gene (Tm5NM3, 5, 6, 8, 9, 11) are viable whereas the failure to generate viable stem cells lacking exon $9 \mathrm{~d}$ of the TPM3 gene (Tm5NM1,2) indicates that at least one of these isoforms is essential for cell growth in vitro (Hook et al. 2011). Their role in cell growth is further supported by the fact that these isoforms are expressed in most, if not all cells and there is an increased reliance on these isoforms in almost all forms of cancer (Stehn et al. 2006). These results demonstrate that isoforms from the TPM3 gene are required for the normal functioning of a cell and cannot be compensated for by products from the other three genes.

Excitation contraction coupling in skeletal muscle fibers

The importance of cytoskeletal tropomyosin isoforms in cellular function is seen in vivo in skeletal muscle fibers. In muscles from mice null for Tm5NM1 the level of T-tubule dysmorphology was increased when compared to WT muscles, and the Tm5NM1 KO mouse muscles had altered contractile properties which were not due to fiber-type changes (Vlahovich et al. 2009). Further experiments revealed the altered contractile performance was a result of dysregulation of T-tubule function due to the loss of Tm5NM1. This demonstrates that the LMW Tm4 expressed in an adjacent location in muscle cannot compensate for the loss of Tm5NM1.

\section{Conclusion}

The specialized function of tropomyosin isoforms described here provides clear evidence that tropomyosin has a much underappreciated role in the functional regulation of the actin cytoskeleton. In light of this, it would seem highly likely that the binding of individual tropomyosin isoforms to actin filaments confers specific functional properties upon these filaments. This gives rise to distinct filament populations localized to specific regions of a cell where they perform different functions.

\section{Mechanisms of specialized tropomyosin function}

Historically, tropomyosin has been both studied and understood in terms of its ability to regulate the myosin II interaction with the actin filament in muscle (Murray and Weber 1973). In vitro studies revealed that chicken gizzard tropomyosin displays a greater cooperativity than rabbit skeletal tropomyosin in terms of their effects on myosin subfragment 1 activity (Lehrer and Morris 1984). Additional protein chemistry studies also revealed that muscle tropomyosin could regulate muscle actin filament stability (Fujime and Ishiwata 1971) and inhibit both DNase 1 
(Hitchcock et al. 1976) and cofilin (Bernstein and Bamburg 1982) induced depolymerisation of muscle actin. The implications of this work for the functional diversity of the cytoskeleton required the development of molecular genetic approaches to manipulate the composition of the cytoskeleton and visualization of different filament populations.

\section{Evolutionary consequences of isoform sorting}

The ability to sort isoforms to different spatial, and therefore functional contexts, will inevitably result in the divergence of their functional capacities due to the differing functional constraints placed upon them. This sorting will lead to the specialized functions of intracellular sites regulated by the specific isoform population present. There is a clear lack of functional redundancy seen between different tropomyosin isoforms, and they show finely tuned spatial segregation to specific subcellular locations (Martin and Gunning 2008). This suggests that the creation of different tropomyosin isoforms throughout evolution allows for the creation of a range of actin filament populations which possess the structural information required to fulfill a broad range of unique functions (Gunning et al. 2008).

\section{Regulate actin polymer levels}

A number of studies have demonstrated the ability of tropomyosin to regulate levels of F-actin within cells. Schevzov et al. (2008) found that following the overexpression of Tm3 and Tm5NM1, the levels of other cytoskeletal tropomyosin isoforms and $\beta$ - and $\gamma$-actin levels were unchanged in transgenic tissues. Interestingly in primary hippocampal neurons from Tm5NM1 transgenic mice, enrichment of Tm5NM1 staining in the growth cones was associated with a significant increase in both total phalloidin signal and mean pixel intensity (Schevzov et al. 2008). Similar results were reported in osteoclasts where manipulation of Tm4 expression resulted in changes in F-actin levels at the site of Tm4 localization. Overexpression of Tm4 caused an increase in F-actin in podosomes, whereas the knockdown of this isoform resulted in significant thinning of $\mathrm{F}$-actin in the actin ring and sealing zone (McMichael and Lee 2008). These findings indicate that the levels of cytoskeletal tropomyosin are limiting for actin polymerization in the subcellular regions where these isoforms are sorted. Thus, tropomyosin isoform sorting regulates total actin polymer levels at specific intracellular locations.

\section{Regulate myosin motors}

Whilst the regulation of myosin driven contraction by tropomyosin has been extensively studied in striated muscle, comparatively little is known about the interactions between tropomyosin dependent regulation of actomyosin interactions in cytoskeletal systems. Work by Fanning et al. (1994) illustrated that the ability of tropomyosin to regulate the ATPase activity and translocation of muscle myosin II along actin filaments was dependent on the isoform present. In contrast, all tropomyosin isoforms tested inhibited the ATPase activity and translocation of myosin I to a similar extent. This demonstrated that regulation of myosin motor interactions with actin was isoform dependent and that a single isoform can have opposing effects on different myosin motors.

In fission yeast the tropomyosin isoform Cdc8p was found to enhance myosin II motor activity, promoting the formation of the contractile ring (Stark et al. 2010). Further work by Lord's laboratory has shown that Cdc8p also regulates the activity of myosin V (Clayton et al. 2010). Coulton et al. (2010) found that whilst acetylation of Cdc8p was required for the regulation of myosin II, there was no effect on the regulation of myosin I or V. Recent work has demonstrated that tropomyosin allows for the processive movement of class $\mathrm{V}$ myosins in $S$. cerevisiae. Hodges et al. (2012) found whilst class V myosin motors are unable to move processively along bare skeletal muscle actin, supporting previous in vitro studies, the creation of a more biologically relevant filament through the addition of tropomyosin allowed for the processive movement of Myo2p. This data strongly supports the idea that actomyosin interactions are sensitive to the presence of tropomyosin along the filament.

The regulation of myosin has also been seen in more complex mammalian cells. Bryce et al. (2003) found that myosin IIA, but not IIB, was recruited to stress fibers in Tm5NM1 overexpressing B35 cells resulting in a substantial increase in myosin II activity. The recruitment of myosin IIA was also seen in the dendrites of cortical neurons from transgenic mice overexpressing Tm5NM1 (Bryce et al. 2003). However, in growth cones where myosin IIA is absent, IIB was able to associate with Tm5NM1 containing filament bundles demonstrating that the preferential recruitment of myosin is dependent upon the availability of specific myosin isoforms (Schevzov et al. 2005a). Tang and Ostap (2001) provided further evidence for the regulation of myosin I by tropomyosin. They found that the exclusion of myosin I from actin structures that contain tropomyosin was due to the regulation of the actomyosin interaction by tropomyosin.

Regulate interactions of other actin binding proteins

\section{Actin depolymerizing factor/cofilin}

Actin depolymerizing factor (ADF)/cofilin depolymerizes actin filaments and was initially found to compete with 
tropoymosin for binding to the filament (Bernstein and Bamburg 1982). Bryce et al. (2003) found that this antagonistic interaction was isoform specific. Tm5NM1 expressing cells had an increased level of phosphorylated ADF indicating that it was displaced from actin filaments, however $\mathrm{TmBr} 3$ recruits $\mathrm{ADF}$ to the lamellapodia where they colocalize on the same filaments. Thus, tropomyosins can be seen as collaborators or competitors of ADF/cofilin depending on the tropomyosin isoform (Kuhn and Bamburg 2008).

\section{Fascin}

Fascin is an actin bundling protein found in stress fibers and filipodia which localizes with HMW but not LMW tropomyosin isoforms (Yamashiro-Matsumura and Matsumura 1986). In control B35 neuroblastoma cells there is an association of fascin with Tm2. Creed et al. (2011) found that in $\mathrm{Tm} 3$ overexpressing cells there was a significant shift in fascin association from Tm2 to Tm3containing filaments. Furthermore they found that the overexpression of $\mathrm{Tm} 3$ resulted in an increase in fascin expression. This indicates that different tropomyosin isoforms can alter the expression of endogenous actin binding proteins, possibly via changing the partitioning of these proteins between the soluble and filament bound pools and hence their turnover kinetics.

\section{Formin homology proteins}

Formins are a diverse family of actin nucleating proteins. Whilst there have been several biochemical studies which investigate the kinetics of the interaction between formins and tropomyosin (Wawro et al. 2007; Ujfalusi et al. 2009, 2012), there has been little work describing this interaction in a cell based system. In fission yeast, the formin $\mathrm{Cdc} 12 \mathrm{p}$ nucleates actin filaments which the tropomyosin isoform Cdc8p binds to with diverse effects on Cdc12p-mediated actin assembly (Skau et al. 2009). Cdc8p's binding both increases the rate of elongation and allows annealing of the filaments before stopping Cdc12p-mediated elongation. Interestingly, Cdc8p may then stop Cdc12p-mediated elongation through either the trapping of Cdc12p or dissociating it from the filament (Skau et al. 2009). This intricate relationship is still not fully understood and it is likely that the interactions which occur in mammalian cells, which express multiple formin and tropomyosin isoforms, would be far more complex. Whilst the consequences of this are not known, this study suggests that tropomyosin's role in the regulation of the cytoskeleton is far more complex and intricate than previously thought.
Responsive to availability of active ABPs

More recently it was shown that the HMW isoform Tm3 independently regulates the function of actin filaments at specific intracellular sites (Creed et al. 2011). They found that active ADF/cofilin was localized in the cell body and within the base of the filipodia in Tm3 overexpressing cells, whilst the inactive phosphorylated ADF/cofilin was only found in the perinuclear region. This indicates that only the active ADF/cofilin is able to be recruited to Tm3containing filaments. Furthermore they demonstrated that inactivation or knockdown of ADF/cofilin caused a change in cell morphology and cytoskeletal organization more resembling the control B35 cells (Creed et al. 2011). This indicates that whilst different tropomyosin isoforms will preferentially recruit specific ABPs, their impact on the cell is also determined by the local availability of active ABP's.

\section{The actin-tropomyosin copolymer as the unit of function}

Early research into understanding the cytoskeleton was dominated by protein biochemistry and in vitro studies, which have been critical in providing a basis for in vivo studies. However, whilst in vitro work has been fundamental in establishing many of the key concepts known today and has driven the model building surrounding interactions of cytoskeletal proteins, there have been numerous cases where the biochemical data has not been supported by molecular genetics. Interpretation of in vitro observations has established a view that filaments are generic and the specific functional outcomes observed within cells are due to the chance interaction of a large number of actin binding proteins with filament bundles at any given time. However, many of these experiments were performed in a fixed environment, under ideal conditions using $\alpha$-skeletal, not cytoskeletal actin and in the absence of actin binding proteins needed to assemble filaments into the biological structures seen in vivo. In contrast, molecular genetics and in vivo data have driven functional biology. The sorting of actin binding proteins gives rise to filament bundles with unique functional information that is critical for the functioning of a cell.

Whilst many biochemical studies have demonstrated both polymerization of actin and subsequent binding of cytoskeletal actin binding proteins to skeletal actin in vitro, how these processes occur in vivo is yet to be established. There is an ever increasing body of evidence to suggest that there is no active transport for the movement of tropomyosin to specific spatial regions, and that its sorting relies on the active formation of filaments. This suggests a 'molecular sink' model whereby isoforms accumulate in structures 
A

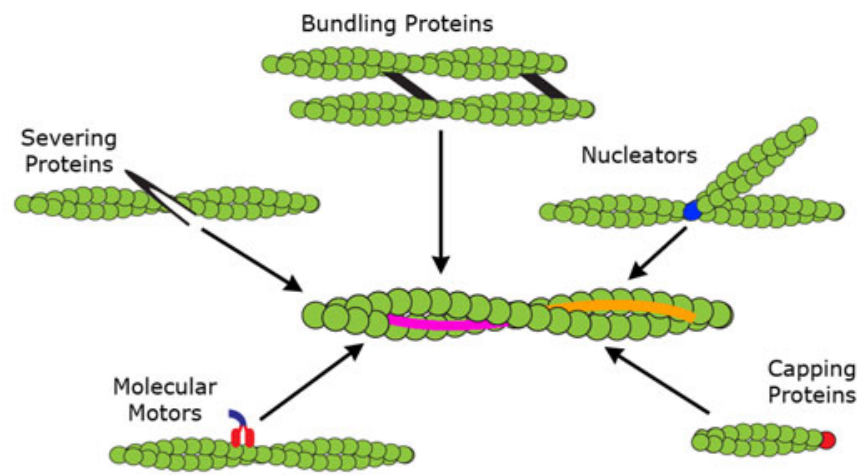



Fig. 3 a Previous view: Actin filaments are formed independent of any association with actin binding proteins aside from their nucleators. Once formed tropomyosin isoforms nonspecifically dimerize with any isoform of the same molecular weight. At a critical concentration these homo- or heterodimers will form heteropolymers along the filament where they act only to stabilize the actin filament. b Current model: Actin filaments are nucleated and begin to polymerize. Specific tropomyosin isoforms co-polymerize with the newly nucleated filaments stabilizing them whilst polymerization continues. Once the mature filament has formed the bound tropomyosin regulates the interaction between actin and actin binding

where they are most stable. This hypothesis has been supported by drug studies demonstrating that a loss of filaments results in the abolishment of isoform sorting. Intrinsic to the sorting of tropomyosin is that tropomyosin binds to polymerizing actin filaments, both providing a mechanism of stabilizing single filaments and facilitating the interactions with surrounding actin binding proteins.

Whilst the notion of gestalt-binding proposed by Holmes and Lehman (2008) would accurately predict the binding of tropomyosin to actin filaments in vitro, it fails to take into account the complexity of the cellular environment. The formation of filaments in a cellular environment is a continuous process that occurs throughout the whole

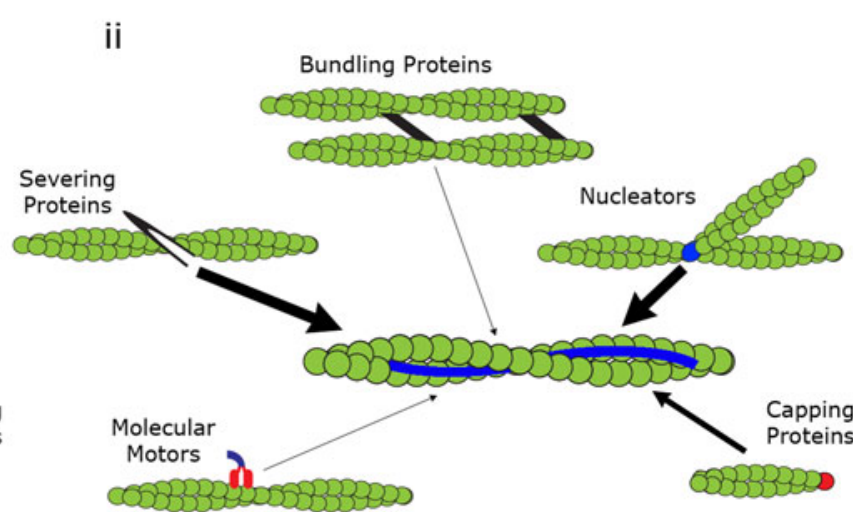

proteins. (i) Seen above the red decorated filaments there is enhanced binding of bundling proteins and molecular motors, whilst nucleators and capping protein interaction is greatly diminished. There is also complete exclusion of severing proteins from these structures. (ii) In contrast, filaments decorated by blue tropomyosin enhance the severing protein's binding whilst modulating the access of other actin binding proteins independent of the red filaments. This allows for the formation of multiple filament populations which are able to perform specific cellular functions and that are independently regulated in different regions of the cell

cell. Therefore, the notion that an actin filament forms in an environment free from interactions with the plethora of actin binding proteins present throughout the cytoplasm would seem a highly inefficient process. On the other hand, the binding of specific tropomyosin isoforms to nucleated actin filaments whose conformational twist favors that particular isoform, would allow not only for the stabilization of the growing filament, but for the formation of a functionally distinct filament population already equipped with the structural information required to fulfill a specific functional role. Given the dynamic nature of actin filaments in an intracellular environment (for example, see Tojkander et al. 2011) it seems more likely that specific 
actin and tropomyosin isoforms co-assemble to form functionally distinct filaments. The mechanism of assembly may be influenced by the nature of filament nucleation in a cell which differs from that used in most in vitro studies.

The proposal that tropomyosin plays a somewhat redundant role in cytoskeletal regulation where its binding can be so easily disrupted by other binding proteins is questioned by the presence of a number of essential tropomyosin isoforms within the cell. The finely regulated sorting of tropomyosin to distinct filament populations, highly regulated both in time and space would indicate that its role is far more important than a 'parking attendant' for actin filaments. Instead the overwhelming accumulation of data now proposes a more biologically relevant model for the tropomyosin dependent regulation of the actin cytoskeleton (Gunning et al. 2008).

From the results discussed in this review, it would seem obvious in retrospect, that tropomyosin must play a key role in the regulation of the actin cytoskeleton. The two cytoskeletal actin isoforms; $\beta$ - and $\gamma$-actin vary by only a few amino acids, presumably as further changes to the highly conserved amino acid sequence would result in an inability to provide adequate structural support. In contrast, there are over 40 tropomyosin isoforms that are extensively regulated both spatially and temporally. The differential sorting of these structurally distinct isoforms to specific subcellular locations allows for the formation of functionally distinct actin filament populations, each possessing unique structural information.

Here the various tropomyosin isoforms act primarily as choreographers regulating the dynamic interaction between actin and all its binding proteins. This dynamic system gives rise to the formation of unique and functionally distinct filament systems largely based on one gene family. From an evolutionary perspective, cells which were able to utilize multiple filament systems independently regulated by single tropomyosin isoforms would be given a distinct advantage. This would likely have driven the evolution of the tropomyosin dependent regulation of the actin cytoskeleton and the basis for the functional diversity that has become characteristic of the actin filament system. This is described graphically in Fig. 3.

Acknowledgments We'd like to thank Dr. Justine Stehn and Ms. Ashleigh Swain for critical reading of this manuscript. This work has been supported by two funding bodies; generous donations from The Kids Cancer Project and a National Health and Medical Research Council (NHMRC) Project Grant (APP1004188). Howard Vindin is a recipient of an Australian Postgraduate Award.

Open Access This article is distributed under the terms of the Creative Commons Attribution License which permits any use, distribution, and reproduction in any medium, provided the original author(s) and the source are credited.

\section{References}

Balasubramanian MK, Helfman DM, Hemmingsen SM (1992) A new tropomyosin essential for cytokinesis in the fission yeast $S$. pombe. Nature 360(6399):84-87

Bernstein BW, Bamburg JR (1982) Tropomyosin binding to F-actin protects the F-actin from disassembly by brain actin-depolymerizing factor (ADF). Cell Motil 2(1):1-8. doi:10.1002/cm.970020102

Blanchard EM, Iizuka K, Christe M, Conner DA, GeisterferLowrance A, Schoen FJ, Maughan DW, Seidman CE, Seidman JG (1997) Targeted ablation of the murine $\alpha$-tropomyosin gene. Circ Res 81(6):1005-1010. doi:10.1161/01.res.81.6.1005

Bryce NS, Schevzov G, Ferguson V, Percival JM, Lin JJ-C, Matsumura F, Bamburg JR, Jeffrey PL, Hardeman EC, Gunning P, Weinberger RP (2003) Specification of actin filament function and molecular composition by tropomyosin isoforms. Mol Biol Cell 14(3):1002-1016. doi:10.1091/mbc.E02-04-0244

Burgoyne RD, Norman K-M (1985a) Immunocytochemical localization of tropomyosin in rat cerebellum. Brain Res 361(1-2): 178-184. doi:10.1016/0006-8993(85)91287-9

Burgoyne RD, Norman K-M (1985b) Presence of tropomyosin in adrenal chromaffin cells and its association with chromaffin granule membranes. FEBS Lett 179(1):25-28. doi:10.1016/ 0014-5793(85)80183-6

Clayton JE, Sammons, Stark BC, Hodges AR, Lord M (2010) Differential regulation of unconventional fission yeast myosins via the actin track. Curr Biol 20(16):1423-1431. doi:10.1016/ j.cub.2010.07.026

Cooley BC, Bergtrom G (2001) Multiple combinations of alternatively spliced exons in rat tropomyosin- $\alpha$ gene MRNA: evidence for 20 new isoforms in adult tissues and cultured cells. Arch Biochem Biophys 390(1):71-77. doi:10.1006/abbi.2001.2347

Coulton AT, East DA, Galinska-Rakoczy A, Lehman W, Mulvihill DP (2010) The recruitment of acetylated and unacetylated tropomyosin to distinct actin polymers permits the discrete regulation of specific myosins in fission yeast. J Cell Sci 123(19): 3235-3243. doi:10.1242/jcs.069971

Creed SJ, Desouza M, Bamburg JR, Gunning P, Stehn J (2011) Tropomyosin isoform 3 promotes the formation of filopodia by regulating the recruitment of actin-binding proteins to actin filaments. Exp Cell Res 317(3):249-261. doi:10.1016/j.yexcr. 2010.10.019

Dalby-Payne JR, O’Loughlin EV, Gunning P (2003) Polarization of specific tropomyosin isoforms in gastrointestinal epithelial cells and their impact on CFTR at the apical surface. Mol Biol Cell 14(11):4365-4375. doi:10.1091/mbc.E03-03-0169

Drees B, Brown C, Barrell BG, Bretscher A (1995) Tropomyosin is essential in yeast, yet the TPM1 and TPM2 products perform distinct functions. J Cell Biol 128(3):383-392. doi:10.1083/jcb. 128.3.383

Dufour C, Weinberger RP, Schevzov G, Jeffrey PL, Gunning P (1998) Splicing of two internal and four carboxyl-terminal alternative exons in nonmuscle tropomyosin 5 pre-mRNA is independently regulated during development. J Biol Chem 273(29):18547-18555. doi:10.1074/jbc.273.29.18547

Eppinga RD, Li Y, Lin JLC, Lin JJC (2006) Tropomyosin and caldesmon regulate cytokinesis speed and membrane stability during cell division. Arch Biochem Biophys 456(2):161-174. doi:10.1016/j.abb.2006.06.015

Fanning AS, Wolenski JS, Mooseker MS, Izant JG (1994) Differential regulation of skeletal muscle myosin-II and brush border myosin-I enzymology and mechanochemistry by bacterially produced tropomyosin isoforms. Cell Motil Cytoskelet 29(1):29-45. doi:10.1002/cm.970290104 
Fath T, Agnes Chan Y-K, Vrhovski B, Clarke H, Curthoys N, Hook J, Lemckert F, Schevzov G, Tam P, Watson CM, Khoo P-L, Gunning P (2010) New aspects of tropomyosin-regulated neuritogenesis revealed by the deletion of Tm5NM1 and 2 . Eur J Cell Biol 89(7):489-498. doi:10.1016/j.ejcb.2009.11.028

Fujime S, Ishiwata S (1971) Dynamic study of F-actin by quasielastic scattering of laser light. J Mol Biol 62(1):251-265

Gallant C, Appel S, Graceffa P, Leavis P, Lin JJ-C, Gunning PW, Schevzov G, Chaponnier C, DeGnore J, Lehman W, Morgan KG (2011) Tropomyosin variants describe distinct functional subcellular domains in differentiated vascular smooth muscle cells. Am J Physiol Cell Physiol 300(6):1356-1365. doi:10.1152/ ajpcell.00450.2010

Gunning P, Hardeman E, Jeffrey P, Weinberger R (1998a) Creating intracellular structural domains: spatial segregation of actin and tropomyosin isoforms in neurons. BioEssays 20:892-900

Gunning P, Weinberger R, Jeffrey P, Hardeman E (1998b) Isoform sorting and the creation of intracellular compartments. Annu Rev Cell Dev Biol 14:339-372

Gunning PW, Schevzov G, Kee AJ, Hardeman EC (2005) Tropomyosin isoforms: divining rods for actin cytoskeleton function. Trends Cell Biol 15(6):333-341. doi:10.1016/j.tcb.2005.04.007

Gunning P, O’Neill G, Hardeman E (2008) Tropomyosin-based regulation of the actin cytoskeleton in time and space. Physiol Rev 88(1):1-35. doi:10.1152/physrev.00001.2007

Had L, Faivre-Sarrailh C, Legrand C, Mery J, Brugidou J, Rabie A (1994) Tropomyosin isoforms in rat neurons: the different developmental profiles and distributions of $\mathrm{TM}-4$ and $\mathrm{TMBr}-3$ are consistent with different functions. J Cell Sci 107(10):2961-2973

Hannan AJ, Schevzov G, Gunning P, Jeffrey PL, Weinberger RP (1995) Intracellular localization of tropomyosin mRNA and protein is associated with development of neuronal polarity. Mol Cell Neurosci 6(5):397-412

Hannan AJ, Gunning P, Jeffrey PL, Weinberger RP (1998) Structural compartments within neurons: developmentally regulated organization of microfilament isoform mRNA and protein. Mol Cell Neurosci 11(5-6):289-304

Heimann K, Percival JM, Weinberger R, Gunning P, Stow JL (1999) Specific isoforms of actin-binding proteins on distinct populations of golgi-derived vesicles. J Biol Chem 274(16):10743-10750

Herman IM (1993) Actin isoforms. Curr Opin Cell Biol 5(1):48-55. doi:10.1016/S0955-0674(05)80007-9

Hitchcock SE, Carisson L, Lindberge U (1976) Depolymerization of F-actin by deoxyribonuclease I. Cell 7(4):531-542

Hodges Alex R, Krementsova Elena B, Bookwalter Carol S, Fagnant Patricia M, Sladewski Thomas E, Trybus Kathleen M (2012) Tropomyosin is essential for processive movement of a class $\mathrm{V}$ myosin from budding yeast. Curr Biol 22(15):1410-1416. doi:10.1016/j.cub.2012.05.035

Holmes K, Lehman W (2008) Gestalt-binding of tropomyosin to actin filaments. J Muscle Res Cell Motil 29(6-8):213-219. doi:10. 1007/s10974-008-9157-6

Hook J, Lemckert F, Qin H, Schevzov G, Gunning P (2004) Gamma tropomyosin gene products are required for embryonic development. Mol Cell Biol 24(6):2318-2323. doi:10.1128/mcb.24.6. 2318-2323.2004

Hook J, Lemckert F, Schevzov G, Fath T, Gunning P (2011) Functional identity of the gamma tropomyosin gene: implications for embryonic development, reproduction and cell viability. BioArch 1(1):49-59

Hughes JAI, Cooke-Yarborough CM, Chadwick NC, Schevzov G, Arbuckle SM, Gunning P, Weinberger RP (2003) High-molecular-weight tropomyosins localize to the contractile rings of dividing CNS cells but are absent from malignant pediatric and adult CNS tumors. Glia 42(1):25-35. doi:10.1002/glia.10174
Huxley HE (1973) Structural changes in the actin- and myosincontaining filaments during contraction. Cold Spring Harbor Symp Quant Biol 37:361-376. doi:10.1101/sqb.1973.037.01.046

Jagatheesan G, Rajan S, Wieczorek DF (2010) Investigations into tropomyosin function using mouse models. J Mol Cell Cardiol 48(5):893-898. doi:10.1016/j.yjmcc.2009.10.003

Kee AJ, Schevzov G, Nair-Shalliker V, Robinson CS, Vrhovski B, Ghoddusi M, Qiu MR, Lin JJ-C, Weinberger R, Gunning PW, Hardeman EC (2004) Sorting of a nonmuscle tropomyosin to a novel cytoskeletal compartment in skeletal muscle results in muscular dystrophy. J Cell Biol 166(5):685-696. doi:10.1083/ jcb.200406181

Kee A, Gunning P, Hardeman E (2009) Diverse roles of the actin cytoskeleton in striated muscle. J Muscle Res Cell Motil 30: 187-197. doi:10.1007/s10974-009-9193-x

Kotadiya P, McMichael BK, Lee BS (2008) High molecular weight tropomyosins regulate osteoclast cytoskeletal morphology. Bone 43(5):951-960. doi:10.1016/j.bone.2008.06.017

Kuhn T, Bamburg J (2008) Tropomyosin and ADF/Cofilin as Collaborators and Competitors. In: Gunning P (ed) Tropomyosin, vol 644. Adv Exp Med Biol. Springer, New York, pp 232-249

Lehrer SS, Morris EP (1984) Comparison of the effects of smooth and skeletal tropomyosin on skeletal actomyosin subfragment 1 ATPase. J Biol Chem 257(14):8073-8080

Lin JJ, Hegmann TE, Lin JL (1988) Differential localization of tropomyosin isoforms in cultured nonmuscle cells. J Cell Biol 107(2):563-572. doi:10.1083/jcb.107.2.563

Liu H, Bretscher A (1989) Disruption of the single tropomyosin gene in yeast results in the disappearance of actin cables from the cytoskeleton. Cell 57(2):233-242

Martin C, Gunning P (2008) Isoform sorting of tropomyosins. In: Gunning P (ed) Tropomyosin, vol 644. Adv Exp Med Biol. Springer, New York, pp 187-200. doi:10.1007/978-0-387-85766-4_15

Martin C, Schevzov G, Gunning P (2010) Alternatively spliced $\mathrm{N}$-terminal exons in tropomyosin isoforms do not act as autonomous targeting signals. J Struct Biol 170(2):286-293

McMichael BK, Lee BS (2008) Tropomyosin 4 regulates adhesion structures and resorptive capacity in osteoclasts. Exp Cell Res 314(3):564-573. doi:10.1016/j.yexcr.2007.10.018

McMichael BK, Kotadiya P, Singh T, Holliday LS, Lee BS (2006) Tropomyosin isoforms localize to distinct microfilament populations in osteoclasts. Bone 39(4):694-705

Murray JM, Weber A (1973) Molecular control mechanisms in muscle contraction. Physiol Rev 53:612-673

Muthuchamy M, Grupp IL, Grupp G, O’Toole BA, Kier AB, Boivin GP, Neumann J, Wieczorek DF (1995) Molecular and physiological effects of overexpressing striated muscle $\beta$-tropomyosin in the adult murine heart. J Biol Chem 270(51):30593-30603. doi:10.1074/jbc. 270.51 .30593

Muthuchamy M, Boivin GP, Grupp IL, Wieczorek DF (1998) $\beta$-Tropomyosin overexpression induces severe cardiac abnormalities. J Mol Cell Cardiol 30(8):1545-1557. doi:10.1006/jmcc. 1998.0720

Palmiter KA, Kitada Y, Muthuchamy M, Wieczorek DF, Solaro RJ (1996) Exchange of $\alpha$-for $\beta$-tropomyosin in hearts of transgenic mice induces changes in thin filament response to $\mathrm{Ca}$, strong cross-bridge binding, and protein phosphorylation. J Biol Chem 271(20):11611-11614. doi:10.1074/jbc.271.20.11611

Pelham RJ, Lin JJ, Wang YL (1996) A high molecular mass nonmuscle tropomyosin isoform stimulates retrograde organelle transport. J Cell Sci 109(5):981-989

Percival JM, Thomas G, Cock T-A, Gardiner EM, Jeffrey PL, Lin JJC, Weinberger RP, Gunning P (2000) Sorting of tropomyosin isoforms in synchronised NIH 3T3 fibroblasts: evidence for distinct microfilament populations. Cell Motil Cytoskelet 
47(3):189-208. doi:10.1002/1097-0169(200011)47:3<189:aid$\mathrm{cm} 3>3.0 . \mathrm{co} ; 2-\mathrm{c}$

Percival JM, Hughes JAI, Brown DL, Schevzov G, Heimann K, Vrhovski B, Bryce N, Stow JL, Gunning PW (2004) Targeting of a tropomyosin isoform to short microfilaments associated with the golgi complex. Mol Biol Cell 15(1):268-280. doi:10.1091/ mbc.E03-03-0176

Pittenger MF, Kazzaz JA, Helfman DM (1994) Functional properties of non-muscle tropomyosin isoforms. Curr Opin Cell Biol 6(1):96-104. doi:10.1016/0955-0674(94)90122-8

Pruyne D (2008) Tropomyosin function in yeast. Adv Exp Med Biol 664:168-186

Rethinasamy P, Muthuchamy M, Hewett T, Boivin G, Wolska BM, Evans C, Solaro RJ, Wieczorek DF (1998) Molecular and physiological effects of $\alpha$-tropomyosin ablation in the mouse. Circ Res 82(1):116-123. doi:10.1161/01.res.82.1.116

Sanders C, Burtnick LD, Smillie LB (1986) Native chicken gizzard tropomyosin is predominantly a beta gamma-heterodimer. J Biol Chem 261(27):12774-12778

Schevzov G, Gunning P, Jeffrey PL, Temm-Grove C, Helfman DM, Lin JJC, Weinberger RP (1997) Tropomyosin localization reveals distinct populations of microfilaments in neurites and growth cones. Mol Cell Neurosci 8(6):439-454. doi:10.1006/ mone.1997.0599

Schevzov G, Bryce NS, Almonte-Baldonado R, Joya J, Lin JJ-C, Hardeman E, Weinberger R, Gunning P (2005a) Specific features of neuronal size and shape are regulated by tropomyosin isoforms. Mol Biol Cell 16(7):3425-3437. doi:10.1091/mbc. E04-10-0951

Schevzov G, Vrhovski B, Bryce NS, Elmir S, Qiu MR, O’Neill GM, Yang N, Verrills NM, Kavallaris M, Gunning PW (2005b) Tissue-specific tropomyosin isoform composition. J Histochem Cytochem 53(5):557-570. doi:10.1369/jhc.4A6505.2005

Schevzov G, Fath T, Vrhovski B, Vlahovich N, Rajan S, Hook J, Joya JE, Lemckert F, Puttur F, Lin JJ-C, Hardeman EC, Wieczorek DF, O'Neill GM, Gunning PW (2008) Divergent regulation of the sarcomere and the cytoskeleton. J Biol Chem 283(1): 275-283. doi:10.1074/jbc.M704392200

Schevzov G, Whittaker SP, Fath T, Lin JJC, Gunning PW (2011) Tropomyosin isoforms and reagents. BioArch 1(4):135-164

Skau CT, Neidt EM, Kovar DR (2009) Role of tropomyosin in formin-mediated contractile ring assembly in fission yeast. Mol Biol Cell 20(8):2160-2173. doi:10.1091/mbc.E08-12-1201

Skoumpla K, Coulton AT, Lehman W, Geeves MA, Mulvihill DP (2007) Acetylation regulates tropomyosin function in the fission yeast Schizosaccharomyces pombe. J Cell Sci 120(9): 1635-1645. doi:10.1242/jcs.001115

Stark BC, Sladewski TE, Pollard LW, Lord M (2010) Tropomyosin and myosin-ii cellular levels promote actomyosin ring assembly in fission yeast. Mol Biol Cell 21(6):989-1000. doi:10.1091/ mbc.E09-10-0852

Stehn J, Schevzov G, O`Neill G, Gunning P (2006) Specialisation of the tropomyosin composition of actin filaments provides new potential targets for chemotherapy. Curr Cancer Drug Targets 6(3):245-256. doi:10.2174/156800906776842948

Tang N, Ostap EM (2001) Motor domain-dependent localization of myolb (myr-1). Curr Biol 11(14):1131-1135. doi:10.1016/S09609822(01)00320-7

Temm-Grove CJ, Jockusch BM, Weinberger RP, Schevzov G, Helfman DM (1998) Distinct localizations of tropomyosin isoforms in LLC-PK1 epithelial cells suggests specialized function at cell-cell adhesions. Cell Motil Cytoskelet 40(4):393-407

Tojkander S, Gateva G, Schevzov G, Hotulainen P, Naumanen P, Martin C, Gunning Peter W, Lappalainen P (2011) A molecular pathway for myosin ii recruitment to stress fibers. Curr Biol 21(7):539-550

Ujfalusi Z, Vig A, Hild G, Nyitrai M (2009) Effect of tropomyosin on formin-bound actin filaments. Biophys J 96(1):162-168. doi:10. 1529/biophysj.108.138420

Ujfalusi Z, Kovács M, Nagy NT, Barkó S, Hild G, Lukács A, Nyitrai M, Bugyi B (2012) Myosin and tropomyosin stabilize the conformation of formin-nucleated actin filaments. J Biol Chem 287(38):31894-31904. doi:10.1074/jbc.M112.341230

Vlahovich N, Schevzov G, Nair-Shaliker V, Ilkovski B, Artap ST, Joya JE, Kee AJ, North KN, Gunning PW, Hardeman EC (2008) Tropomyosin 4 defines novel filaments in skeletal muscle associated with muscle remodelling/regeneration in normal and diseased muscle. Cell Motil Cytoskelet 65(1):73-85. doi:10. 1002/cm.20245

Vlahovich N, Kee AJ, Van der Poel C, Kettle E, Hernandez-Deviez D, Lucas C, Lynch GS, Parton RG, Gunning PW, Hardeman EC (2009) Cytoskeletal tropomyosin Tm5NM1 is required for normal excitation-contraction coupling in skeletal muscle. Mol Biol Cell 20(1):400-409. doi:10.1091/mbc.E08-06-0616

Wawro B, Greenfield NJ, Wear MA, Cooper JA, Higgs HN, Hitchcock-DeGregori SE (2007) Tropomyosin regulates elongation by formin at the fast-growing end of the actin filament. Biochemistry 46(27):8146-8155. doi:10.1021/bi700686p

Weinberger R, Schevzov G, Jeffrey P, Gordon K, Hill M, Gunning P (1996) The molecular composition of neuronal microfilaments is spatially and temporally regulated. J Neurosci 16(1):238-252

Yamashiro-Matsumura S, Matsumura F (1986) Intracellular localization of the 55-kD actin-bundling protein in cultured cells: spatial relationships with actin, alpha-actinin, tropomyosin, and fimbrin. J Cell Biol 103(2):631-640. doi:10.1083/jcb.103.2.631 